\title{
Tumour versus nephron gene expression yields survival score
}

( omparing tumour genetic heterogeneity to that of the nearby nephron can help to risk-stratify prognosis in patients with renal cell carcinoma (RCC), according to new data presented in European Urology.

Although a number of prognostic models have been proposed in renal cancer, including genetic and epigenetic signatures and pathological scores such as the SSIGN (stage/size/grade/necrosis) risk score, none have been shown to be completely accurate in determining prognosis. Based on the hypothesis that less differentiated clear cell tumours with genetic expression patterns similar to the normal renal cortex or glomerulus display a less aggressive phenotype, Büttner et al. investigated whether the degree of differentiation of RCC from the normal renal tissue in the nephron might be associated with cancer-specific survival (CSS).

The researchers, who hail from a number of institutions across Germany, collected gene expression data of 479 clear cell RCCs (ccRCCs), 212 papillary RCCs (pRCCs), and 66 chromophobe RCCs (chRCCs) from The Cancer Genome Atlas (TCGA), and correlated them to the expression data of nephron cell types. The different tumour types are thought to arise from different areas of the nephron: ccRCC and pRCC most likely originate from the proximal tubules, whereas chRCC is thought to arise in the distal nephron. Analysis of gene expression profiles from eight nephron regions (glomeruli, initial/terminal part of proximal tubule, medullary/cortical thick ascending limbs of the loop of Henle, distal convoluted tubules, cortical/outer medullary collecting ducts) identified 97 genes that made it possible for the team to distinguish different areas of the nephron. Expression levels of these genes were then correlated to expression data of tumours of all three different RCC subtypes.

Expression levels in the majority of ccRCC and pRCC samples generally displayed a higher correlation to the proximal than distal parts of the nephron, as expected, and gene expression in chRCC appeared more similar to the distal parts.

Subsequent cluster analyses identified two clusters for ccRCC, pRCC, and chRCC, which were associated with significant differences in CSS for the ccRCC and pRCC subgroups. Gene expression profiles of ccRCCs and pRCCs and the proximal tubules were more similar in tumours considered to have a better prognosis than in those with estimated poor CSS.

Based on the observation that the main differences between gene expression in clear cell tumours and the proximal tubules were observed in the S1 and S3 regions, Büttner and colleagues created a novel prognostic score, comprising $\mathrm{z}$ scores between the ccRCC samples and the $\mathrm{S} 3$ region, and stratified patients into two groups: those with a high S3-score, indicating high similarity to gene expression in the proximal tubules, and those with a low S3-score. Patients with a high S3-score had better CSS than

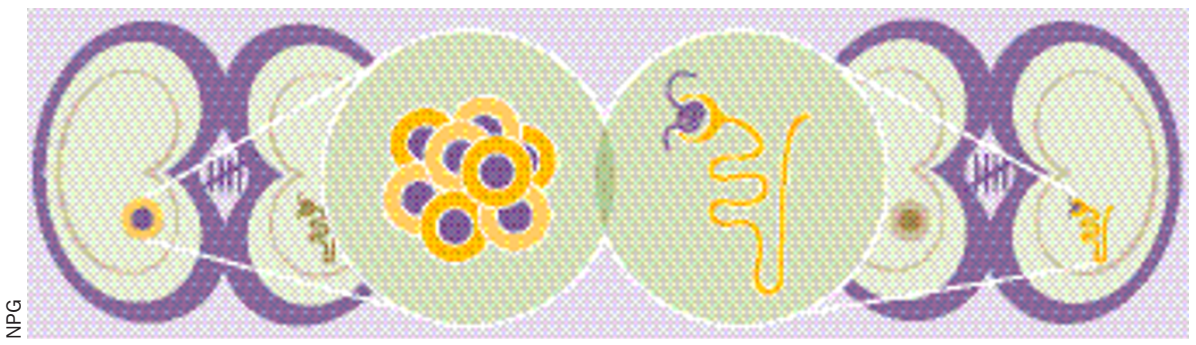

\section{4} had better CSS than those with low S3-scores... 77

those with low S3-scores (HR 3.9, 95\% CI 2.6-5.7; $\left.P_{[\text {log rank }]}=5.3 \mathrm{e}^{-13}\right)$. Patients in the low-S3 group with poor survival also exhibited a higher incidence of advanced, necrotic and metastatic tumours.

Furthermore, the S3-score also enabled prediction of CSS specifically in metastatic (HR 2.3, 95\% CI 1.3-3.9; $P_{[\text {log rank }]}=2.2 \mathrm{e}^{-03}$ ) and nonmetastatic tumours (HR 5.4, 95\% CI 3.0-9.9; $\left.P_{[\text {log rank] }}=9.2 \mathrm{e}^{-10}\right)$.

The team went on to compare the accuracy of their S3-score with previously validated prediction tools. They found in both univariate and multivariate analyses that the S3-score significantly improved the accuracy of the $\mathrm{ccA} / \mathrm{ccB}$, which is currently considered the best gene expression signature for ccRCC prognostication. In addition, combination with the SSIGN criteria further improved CSS prediction. Validation of the relationship between S3-score and CSS in an independent ccRCC cohort $(n=139)$, using the cut-off values established in the TCGA cohort, showed that the S3-score was also significantly associated with CSS in this group of patients (HR 2.9, 95\% CI $\left.1.7-4.8 ; P_{[\text {log rank }]}=3.1 \mathrm{e}^{-05}\right)$.

"Our ongoing and future research activities regarding the newly established S3-score include adaptation and validation of the S3-score for other subtypes of renal cancer, such as papillary RCC," corresponding author Matthias Schwab told Nature Reviews Urology. "We are also planning a clinical trial to validate the novel S3-score prospectively, and working to elucidate the underlying molecular mechanisms to better understand the pathophysiology of the selected 97 genes in the S3-score for prediction of survival."

Annette Fenner 